\title{
NFRC Spectral Data Library \#2 for use with the WINDOW 4.1 Computer Program
}

\author{
Contact: John Sadlier \\ (510) 486-4291, 486-5605 \\ FAX (510) 486-4089
}

\author{
Energy and Environment Division \\ Building Technologies Program \\ Windows and Daylighting Group \\ Ernest Orlando Lawrence Berkeley National Laboratory \\ 1 Cyclotron Road \\ Berkeley CA, 94720
}

February 1996

DISCLAIMER

\begin{abstract}
This report was prepared as an account of work sponsored by an agency of the United States Government. Neither the United States Government nor any agency thereof, nor any of their employees, makes any warranty, express or implied, or assumes any legal liability or responsibility for the accuracy, completeness, or usefulness of any information, apparatus, product, or process disclosed, or represents that its use would not infringe privately owned rights. Reference herein to any specific commercial product, process, or service by trade name, trademark, manufacturer, or otherwise does not necessarily constitute or imply its endorsement, recommendation, or favoring by the United States Government or any agency thereof. The views and opinions of authors expressed herein do not necessarily state or reflect those of the United States Government or any agency thereof.
\end{abstract}

\section{DISTRIBUTION OF THIS DOCUMENT IS UNLIMITED 35}

This data library was created through a collaborative effort between the individual glass glazing manufacturers and coaters involved, the National Fenestration Rating Council (NFRC), the Primary Glass Manufacturers Council (PGMC), and the Lawrence Berkeley National Laboratory (LBNL). LBNL effort was supported by the Assistant Secretary for Energy Efficiency and Renewable Energy, Office of Building Technologies, Building Systems and Materials Division of the US Department of Energy under Contract No. DE-AC03-76SF00098. 
Recyded Paper 


\title{
NFRC Spectral Data Library \#2 For Use With WINDOW 4.1
}

\author{
February 1996 \\ Contact: John Sadlier \\ Windows and Daylighting Group \\ Lawrence Berkeley National Laboratory \\ Berkeley CA, 94720 \\ (510) 486-4291, 486-5605 \\ FAX (510) 486-4089 FAX
}

This library update contains NFRC approved spectral data for the WINDOW analysis program. This information is an update to the spectral data library that is distributed with WINDOW 4.1 and supersedes the NFRC Spectral Data Library Update of October 1995. The NFRC approval stamp appears in the first line of the spectral data file's header. This stamp changes the "*" sign shown in the WINDOW glass library next to spectral data file names to a "\#" sign. Any unauthorized changes to an NFRC approved file will çause it to revert to displaying the "*" sign in the WINDOW glass library.

Beginning April 1, 1996, NFRC is requiring that ALL simulations (for U-factor, Solar Heat Gain Coefficient and Visible Transmittance) MUST be carried out using NFRC approved spectral data only. Any spectral data file displaying the " $\star$ " sign will be obsolete after that date; only files displaying the "\#" sign may be used.

All spectral files must reside directly in the w4|w4liblspecdat directory to be accessible to WINDOW.

The table on the following page summarizes the effects of the library update on the WINDOW glass library structure. A new glass.dat file is included on the distribution disk. This library list . contains only the NFRC approved files with their most recent ID numbers. Each user may configure their own library to include new NFRC approved data, previous WINDOW data and their own spectral data.

\section{Instructions for Loading the Spectral Data Files}

The spectral data is compressed in a self-extracting archive, spclib02.exe. Uncompressed, the full library contains 628 files and takes approximately 4.1 MB of hard disk space. Uncompressing the data will re-create identical versions of the files contained on the October 1995 Spectral Data Library for users that have installed that version. There is no problem if this library overwrites your previously installed files. To de-compress the data, use one of the following options:

Option 1: To create subdirectories sorting files by manufacturer, copy spclib02.exe to your hard disk and run 
spclib02.exe -d

You will have to move the files to $w 4|w 4 l i b| s p e c d a t$ or an equivalent directory to use them with WINDOW.

2. For no subdirectory structure, copy spclib02.exe to the directory $w 4|w 4 l i b| s p e c d a t$ and run spclib02

This option will put the spectral data files directly in the specified directory. All manufacturers' data will be lumped together in the specdat subdirectory.

\section{Instruction for Loading the New Glass Library, glass.dat}

Also included on the disk is the file glassdat.exe. It is a self-extracting archive containing the file glass.dat only. Copy the file glassdat.exe to the directory $w 4 / w 4 l i b$. Uncompress it by running:

\section{glassdat}

HOWEVER, take care not to overwrite the glass.dat file you have been using all along. A suggestion is to rename that as glass.bak before uncompressing the new file.

Many of the older files have been superseded by new data in this library update (see the table which follows). You may use your own word processor or spreadsheet to concatenate the new glass. dat file with your own glass.dat file, and delete files that have been replaced by newer versions. Or you may re-enter the names of files you wish to use through the WINDOW glass library screen. 
Summary of Current WINDOW Spectral Data Files

\begin{tabular}{|c|l|c|c|l|}
\hline $\begin{array}{c}\text { Reserved } \\
\text { ID \# } \\
\text { Range }\end{array}$ & \multicolumn{1}{|c|}{ Manufacturer } & $\begin{array}{c}\text { NFRC } \\
\text { Approved } \\
\text { Files* }\end{array}$ & $\begin{array}{c}\text { Other } \\
\text { Current } \\
\text { Files** }\end{array}$ & \multicolumn{1}{c|}{$\begin{array}{c}\text { Update Information } \\
\text { (Relative to October 1995) }\end{array}$} \\
\hline $100-199$ & Generic Data & $100-104$ & & All New Files \\
\hline $700-1099$ & AFG Industries & $700-883$ & $1000-1001$ & No Updates \\
\hline $1100-1199$ & DuPont & - & $1100-1116$ & No Updates \\
\hline $1200-1299$ & Monsanto & - & $1200-1224$ & No Updates \\
\hline $1300-1399$ & Ford & $1300-1328$ & $1329-1333$ & No Updates \\
\hline $1400-1499$ & Libbey-Owens-Ford & $1400-1439$ & - & No Updates \\
\hline $1500-1599$ & $\begin{array}{l}\text { Southwall } \\
\text { Technologies }\end{array}$ & $1500-1550$ & - & No Updates \\
\hline $1700-1799$ & Marcon Coatings & $1700-1714$ & - & No Updates \\
\hline $2000-2099$ & Cardinal IG & $2000-2019$ & - & No Updates \\
\hline $2500-2599$ & Courtaulds & $2500-2526$ & - & No Updates \\
& Performance Films & - & - & All New Files \\
\hline $2700-2799$ & $3 M$ Corporation & $2700-2724$ & - & No Updates \\
\hline $3000-3199$ & Guardian & $3100-3104$ & $3000-3099$ & No \\
\hline $4000-4199$ & Interpane & - & $4000-4133$ & No Updates \\
\hline $5000-5299$ & PPG Industries & $5001-5248$ & - & No Updates \\
\hline $6000-6199$ & Viracon & $6000-6146$ & - & No updates \\
\hline
\end{tabular}

*NFRC approved files included only on the NFRC WINDOW Spectral Data Library \#2 disk

**These data files will be obsolete after April 1, 1996. They are the spectral data files included on the original WINDOW 4.1 Spectral Data disk of March 1994. AFG Industries, DuPont and Interpane have products in the original glass.dat library file (included in the glass.dat file on the WINDOW 4.1 Program Disk) that do not have spectral data associated with them. There are no updates to these files, so retain the information from the original glass.dat file if you wish to perform analyses with these products for purposes other than certification according to NFRC procedures. Refer to the WINDOW 4.1 User's Manual and the attached Product Cross Reference List for specific file names. 


\section{Generic Uncoated Glass Products}

These are not measured properties of any particular glass product, but are composed of average spectral data from 3 or more files. For simulation purposes, they are considered equivalent files with those listed under the Description heading. They are intended for use in fenestration systems that are customarily produced with glazings from different manufacturers, when the particular glazing manufacturer is not known.

\begin{tabular}{|c|c|c|c|c|}
\hline 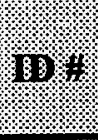 & Tlementine & Gassigpe & 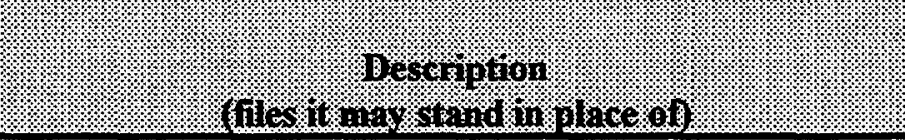 & Whoming \\
\hline 100 & CLEAR 3.DAT & Clear & CLEAR30.FMC, CLEAR3.LOF, CLR-3.CIG, CLEAR 3.PPG & 3.0 \\
\hline 101 & CLEAR 6.DAT & Clear & CLEAR60.FMC, CLEAR6.LOF, CLR-6.CIG, CLEAR 6.PPG & 6.0 \\
\hline 102 & BRONZE 3.DAT & Bronze & BRONZE30.FMC, BRONZ3.LOF, SBRNZ 3.PPG & 3.0 \\
\hline 103 & BRONZE 6.DAT & Bronze & BRONZE60.FMC, BRONZ6.LOF, SBRNZ 6.PPG & 6.0 \\
\hline 104 & GRAY 3.DAT & Gray & GRAY30.FMC, GREY3.LOF, SGRAY 3.PPG & 3.0 \\
\hline
\end{tabular}


AFG Industries, Inc.

\begin{tabular}{|c|c|c|c|c|c|}
\hline 18\% & HIV & 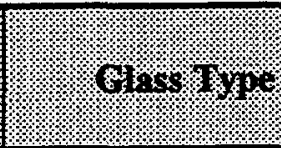 & Souting & 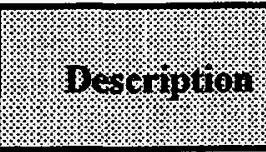 & 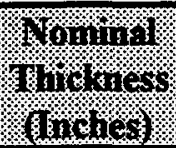 \\
\hline 700 & B120.AFG & Clear & Titanium Nitride & Silver-Blue & $1 / 4^{\prime \prime}$ \\
\hline 701 & B220.AFG & Green & Titanium Nitride & Silver-Blue & $1 / 4^{\prime \prime}$ \\
\hline 702 & B320.AFG & Gray & Titanium Nitride & \begin{tabular}{|l} 
Silver-Blue \\
\end{tabular} & $1 / 4^{\prime \prime}$ \\
\hline 703 & B420.AFG & Bronze & Titanium Nitride & Silver-Blue & $1 / 4^{\prime \prime}$ \\
\hline 704 & B520.AFG & Blue & Titanium Nitride & Silver-Blue & $1 / 4^{\prime \prime}$ \\
\hline 705 & B620.AFG & Blue-green & Titanium Nitride & Silver-Blue & $1 / 4^{\prime \prime}$ \\
\hline 706 & B720.AFG & Azurlite * & Titanium Nitride & Silver-Blue & $1 / 4^{\prime \prime}$ \\
\hline 707 & B820.AFG & Evergreen ** & Titanium Nitride & Silver-Blue & $1 / 4 "$ \\
\hline 708 & B130.AFG & Clear & Titanium Nitride & Silver-Blue & $1 / 4^{\prime \prime}$ \\
\hline 709 & B230.AFG & Green & Titanium Nitride & \begin{tabular}{|l} 
Silver-Blue \\
\end{tabular} & 1/4" \\
\hline 710 & B330.AFG & Gray & Titanium Nitride & Silver-Blue & $1 / 4^{\prime \prime}$ \\
\hline 711 & B430.AFG & Bronze & Titanium Nitride & Silver-Blue & $1 / 4^{\prime \prime}$ \\
\hline 712 & B530.AFG & Blue & Titanium Nitride & Silver-Blue & $1 / 4^{\prime \prime}$ \\
\hline 713 & B630.AFG & Blue-green & Titanium Nitride & \begin{tabular}{|l} 
Silver-Blue \\
\end{tabular} & $1 / 4^{\prime \prime}$ \\
\hline 714 & B730.AFG & Azurlite * & Titanium Nitride & Silver-Blue & $1 / 4^{\prime \prime}$ \\
\hline 715 & B830.AFG & Evergreen ** & Titanium Nitride & Silver-Blue & $1 / 4^{\prime \prime}$ \\
\hline 716 & $\mathrm{~B} 140 . \mathrm{AFG}$ & Clear & Titanium Nitride & Silver-Blue & $1 / 4^{\prime \prime}$ \\
\hline 717 & B240.AFG & Green & Titanium Nitride & Silver-Blue & $1 / 4 "$ \\
\hline 718 & B340.AFG & Gray & Titanium Nitride & Silver-Blue & $1 / 4^{\prime \prime}$ \\
\hline 719 & B440.AFG & Bronze & Titanium Nitride & Silver-Blue & $1 / 4^{\prime \prime}$ \\
\hline 720 & B540.AFG & Blue & Titanium Nitride & \begin{tabular}{|l|} 
Silver-Blue \\
\end{tabular} & $1 / 4^{\prime \prime}$ \\
\hline 721 & B640.AFG & Blue-green & Titanium Nitride & Silver-Blue & $1 / 4^{\prime \prime}$ \\
\hline 722 & B740.AFG & Azurlite * & Titanium Nitride & Silver-Blue & $1 / 4^{\prime \prime}$ \\
\hline 723 & B840.AFG & Evergreen ** & Titanium Nitride & \begin{tabular}{|l} 
Silver-Blue \\
\end{tabular} & $1 / 4^{\prime \prime}$ \\
\hline 724 & C120.AFG & Clear & Copper & Copper & $1 / 4^{\prime \prime}$ \\
\hline 725 & C220.AFG & Green & Copper & Copper & $1 / 4^{\prime \prime}$ \\
\hline 726 & C320.AFG & Gray & Copper & Copper & $1 / 4^{\prime \prime}$ \\
\hline 727 & C420.AFG & Bronze & Copper & Copper & $1 / 4^{\prime \prime}$ \\
\hline 728 & C520.AFG & Blue & Copper & Copper & $1 / 4^{\prime \prime}$ \\
\hline 729 & C620.AFG & Blue-green & Copper & Copper & $1 / 4^{n}$ \\
\hline 730 & C720.AFG & Azurlite * & Copper & Copper & $1 / 4^{\prime \prime}$ \\
\hline 731 & C820.AFG & Evergreen ** & Copper & Copper & $1 / 4^{\prime \prime}$ \\
\hline 732 & C130.AFG & Clear & Copper & Copper & $1 / 4^{\prime \prime}$ \\
\hline 733 & C230.AFG & Green & Copper & Copper & $1 / 4^{\prime \prime}$ \\
\hline 734 & C330.AFG & Gray & Copper & Copper & $1 / 4^{\prime \prime}$ \\
\hline 735 & C430.AFG & Bronze & Copper & Copper & $1 / 4^{\prime \prime}$ \\
\hline 736 & C530.AFG & Blue & Copper & Copper & $1 / 4 "$ \\
\hline 737 & C630.AFG & Blue-green & Copper & Copper & $1 / 4^{\prime \prime}$ \\
\hline 738 & C730.AFG & Azurlite * & Copper & Copper & $1 / 4^{\prime \prime}$ \\
\hline 739 & C830.AFG & Evergreen ** & Copper & Copper & $1 / 4^{\prime \prime}$ \\
\hline 740 & C145.AFG & Clear & Copper & Copper & $1 / 4^{\prime \prime}$ \\
\hline 741 & C245.AFG & Green & Copper & Copper & $1 / 4^{\prime \prime}$ \\
\hline 742 & C345.AFG & Gray & Copper & Copper & $1 / 4^{\prime \prime}$ \\
\hline 743 & C445.AFG & Bronze & Copper & Copper & $1 / 4^{\prime \prime}$ \\
\hline 744 & C545.AFG & Blue & Copper & Copper & $1 / 4^{\prime \prime}$ \\
\hline
\end{tabular}




\begin{tabular}{|c|c|c|c|c|c|}
\hline $41 \%$ & miry & (1. & 604018 & Bescription & Monining \\
\hline 745 & C645.AFG & Blue-green & Copper & Copper & $1 / 4^{\prime \prime}$ \\
\hline 746 & C745.AFG & Azurlite * & Copper & Copper & $1 / 4 "$ \\
\hline 747 & C845.AFG & Evergreen ** & Copper & Copper & 1/4" \\
\hline 748 & C155.AFG & Clear & Copper & Copper & 1/4" \\
\hline 749 & C255.AFG & Green & Copper & Copper & 1/4" \\
\hline 750 & C355.AFG & Gray & Copper & Copper & $1 / 4 "$ \\
\hline 751 & C455.AFG & Bronze & Copper & Copper & 1/4" \\
\hline 752 & C555.AFG & Blue & Copper & Copper & 1/4" \\
\hline 753 & C655.AFG & Blue-green & Copper & Copper & $1 / 4^{\prime \prime}$ \\
\hline 754 & C755.AFG & Azurlite * & Copper & Copper & $1 / 4 "$ \\
\hline 755 & C855.AFG & Evergreen ** & Copper & Copper & I/4" \\
\hline 756 & ES140.AFG & Clear & Silver & Low.\%T Low E & $1 / 4^{\prime \prime}$ \\
\hline 757 & ES240.AFG & Green & Silver & Low.\%T Low E & $1 / 4^{\prime \prime}$ \\
\hline 758 & ES340.AFG & Gray & Silver & Low.\%T Low E & $1 / 4 "$ \\
\hline 759 & ES440.AFG & Bronze & Silver & Low.\%T Low E & $1 / 4^{\prime \prime}$ \\
\hline 760 & ES540.AFG & Blue & Silver & Low.\%T Low E & $1 / 4^{\prime \prime}$ \\
\hline 761 & ES640.AFG & Blue-green & Silver & Low.\%T Low E & $1 / 4^{\prime \prime}$ \\
\hline 762 & ES740.AFG & Azurlite * & Silver & Low.\%T Low E & $1 / 4^{\prime \prime}$ \\
\hline 763 & ES840.AFG & Evergreen ** & Silver & Low.\%T Low E & $1 / 4^{\prime \prime}$ \\
\hline 764 & ES152.AFG & Clear & Silver & Med.\%T Low E & $1 / 4^{\prime \prime}$ \\
\hline 765 & ES252.AFG & Green & Silver & Med.\%T Low E & $1 / 4^{\prime \prime}$ \\
\hline 766 & ES352.AFG & Gray & Silver & Med.\%T Low E & $1 / 4^{\prime \prime}$ \\
\hline 767 & ES452.AFG & Bronze & Silver & Med.\%T Low E & $1 / 4 "$ \\
\hline 768 & ES552.AFG & Blue & Silver & Med.\%T Low E & $1 / 4^{\prime \prime}$ \\
\hline 769 & ES652.AFG & Blue-green & Silver & Med.\%T Low E & $1 / 4^{\prime \prime}$ \\
\hline 770 & ES752.AFG & Azurlite * & Silver & Med.\%T Low E & $1 / 4^{\prime \prime}$ \\
\hline 771 & ES852.AFG & Evergreen ** & Silver & Med.\%T Low E & $1 / 4 "$ \\
\hline 772 & ESB1.AFG & Clear & Silver & Sunbelt Low E & $1 / 4^{\prime \prime}$ \\
\hline 773 & ESB2.AFG & Green & Silver & Sunbelt Low E & $1 / 4 "$ \\
\hline 774 & ESB3.AFG & Gray & Silver & Sunbelt Low E & $1 / 4 "$ \\
\hline 775 & ESB4.AFG & Bronze & Silver & Sunbelt Low E & $1 / 4^{\prime \prime}$ \\
\hline 776 & ESB5.AFG & Blue & Silver & Sunbelt Low E & 1/4" \\
\hline 777 & ESB6.AFG & Blue-green & Silver & Sunbelt Low E & $1 / 4^{\prime \prime}$ \\
\hline 778 & ESB7.AFG & Azurlite * & Silver & Sunbelt Low E & $1 / 4^{\prime \prime}$ \\
\hline 779 & ESB8.AFG & Evergreen ** & Silver & Sunbelt Low E & $1 / 4^{\prime \prime}$ \\
\hline 780 & ESN1.AFG & Clear & Silver & High. \%T Low E & $1 / 4^{\prime \prime}$ \\
\hline 781 & ESN2.AFG & Green & Silver & High. \%T Low E & $1 / 4^{\prime \prime}$ \\
\hline 782 & ESN3.AFG & Gray & Silver & High. \%T Low E & $1 / 4^{\prime \prime}$ \\
\hline 783 & ESN4.AFG & Bronze & Silver & High. \%T Low E & $1 / 4 "$ \\
\hline 784 & ESN5.AFG & Blue & Silver & High. \%T Low E & $1 / 4 "$ \\
\hline 785 & ESN6.AFG & Blue-green & Silver & High. \%T Low E & $1 / 4^{\prime \prime}$ \\
\hline 786 & ESN7.AFG & Azurlite * & Silver & High. \%T Low E & $1 / 4^{\prime \prime}$ \\
\hline 787 & ESN8.AFG & Evergreen ** & Silver & High. \%T Low E & $1 / 4^{\prime \prime}$ \\
\hline 788 & G108.AFG & Clear & Copper Aluminum & Gold & $1 / 4 "$ \\
\hline 789 & G208.AFG & Green & Copper Aluminum & Gold & $1 / 4^{\prime \prime}$ \\
\hline
\end{tabular}


AFG Industries, Inc.

\begin{tabular}{|c|c|c|c|c|c|}
\hline $10 \%$ & ril & 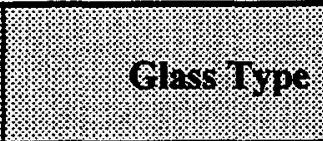 & 8020149 & $84+6+10131$ & 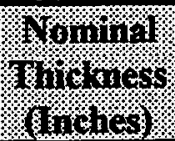 \\
\hline 790 & G308.AFG & Gray & Copper Aluminum & Gold & $1 / 4 "$ \\
\hline 791 & G408.AFG & Bronze & Copper Aluminum & Gold & $1 / 4 "$ \\
\hline 792 & G408.AFG & Blue & Copper Aluminum & Gold & 1/4" \\
\hline 793 & G608.AFG & Blue-green & Copper Aluminum & Gold & $1 / 4 "$ \\
\hline 794 & G708.AFG & Azurlite * & Copper Aluminum & Gold & $1 / 4 "$ \\
\hline 795 & G808.AFG & Evergreen ** & Copper Aluminum & Gold & $1 / 4 "$ \\
\hline 796 & G114.AFG & Clear & Copper Aluminum & Gold & $1 / 4^{\prime \prime}$ \\
\hline 797 & G214.AFG & Green & Copper Aluminum & Gold & $1 / 4 "$ \\
\hline 798 & G314.AFG & Gray & Copper Aluminum & Gold & $1 / 4^{\prime \prime}$ \\
\hline 799 & G414.AFG & Bronze & Copper Aluminum & Gold & $1 / 4^{\prime \prime}$ \\
\hline 800 & G514.AFG & Blue & Copper Aluminum & Gold & $1 / 4 "$ \\
\hline 801 & G614.AFG & Blue-green & Copper Aluminum & Gold & $1 / 4 "$ \\
\hline 802 & G714.AFG & Azurlite * & Copper Aluminum & Gold & $1 / 4 "$ \\
\hline 803 & G814.AFG & Evergreen $* *$ & Copper Aluminum & Gold & $1 / 4 "$ \\
\hline 804 & GP108.AFG & Clear & Stainless Steel & Pewter & $1 / 4^{\prime \prime}$ \\
\hline 805 & GP208.AFG & Green & Stainless Steel & Pewter & 1/4" \\
\hline 806 & GP308.AFG & Gray & Stainless Steel & Pewter & $1 / 4 "$ \\
\hline 807 & GP408.AFG & Bronze & Stainless Steel & Pewter & $1 / 4 "$ \\
\hline 808 & GP508.AFG & Blue & Stainless Steel & Pewter & $1 / 4 "$ \\
\hline 809 & GP608.AFG & Blue-green & Stainless Steel & Pewter & $1 / 4 "$ \\
\hline 810 & GP708.AFG & Azurlite * & Stainless Steel & Pewter & 1/4" \\
\hline 811 & GP808.AFG & Evergreen ** & Stainless Steel & Pewter & $1 / 4 "$ \\
\hline 812 & GP114.AFG & Clear & Stainless Steel & Pewter & $1 / 4 "$ \\
\hline 813 & GP214.AFG & Green & Stainless Steel & Pewter & 1/4" \\
\hline 814 & GP314.AFG & Gray & Stainless Steel & Pewter & $1 / 4^{\prime \prime}$ \\
\hline 815 & GP414.AFG & Bronze & Stainless Steel & Pewter & $1 / 4^{\prime \prime}$ \\
\hline 816 & GP514.AFG & Blue & Stainless Steel & Pewter & $1 / 4^{\prime \prime}$ \\
\hline 817 & GP614.AFG & Blue-green & Stainless Steel & Pewter & 1/4" \\
\hline 818 & GP714.AFG & Azurlite * & Stainless Steel & Pewter & $1 / 4^{\prime \prime}$ \\
\hline 819 & GP814.AFG & Evergreen ** & Stainless Steel & Pewter & $1 / 4 "$ \\
\hline 820 & GP120.AFG & Clear & Stäinless Steel & Pewter & $1 / 4 "$ \\
\hline 821 & GP220.AFG & Green & Stainless Steel & Pewter & $1 / 4^{\prime \prime}$ \\
\hline 822 & GP320.AFG & Gray & Stainless Steel & Pewter & $1 / 4^{\prime \prime}$ \\
\hline 823 & GP420.AFG & Bronze & Stainless Steel & Pewter & $1 / 4^{\prime \prime}$ \\
\hline 824 & GP520.AFG & Blue & Stainless Steel & Pewter & $1 / 4^{\prime \prime}$ \\
\hline 825 & GP620.AFG & Blue-green & Stainless Steel & Pewter & $1 / 4 "$ \\
\hline 826 & GP720.AFG & Azurlite * & Stainless Steel & Pewter & $1 / 4^{\prime \prime}$ \\
\hline 827 & GP820.AFG & Evergreen ** & Stainless Steel & Pewter & $1 / 4 "$ \\
\hline 828 & P120.AFG & Clear & Stainless Steel & Pewter & $1 / 4 "$ \\
\hline 829 & P220.AFG & Green & Stainless Steel & Pewter & $1 / 4 "$ \\
\hline 830 & P320.AFG & Gray & Stainless Steel & Pewter & $1 / 4^{\prime \prime}$ \\
\hline 831 & P420.AFG & Bronze & Stainless Steel & Pewter & $1 / 4^{\prime \prime}$ \\
\hline 832 & P520.AFG & Blue & Stainless Steel & Pewter & $1 / 4^{\prime \prime}$ \\
\hline 833 & P620.AFG & Blue-green & Stainless Steel & Pewter & $1 / 4^{\prime \prime}$ \\
\hline 834 & P720.AFG & Azurlite * & Stainless Steel & Pewter & $1 / 4 "$ \\
\hline
\end{tabular}




\begin{tabular}{|c|c|c|c|c|c|}
\hline 101: & mil 4 Hing & Gass IVP & $1001119 \%$ & Geser 10tion & 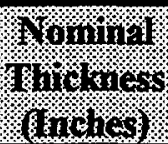 \\
\hline 835 & P820.AFG & Evergreen ** & Stainless Steel & Pewter & $1 / 4^{\prime \prime}$ \\
\hline 836 & P130.AFG & Clear & Stainless Steel & Pewter & $1 / 4^{\prime \prime}$ \\
\hline 837 & P230.AFG & Green & Stainless Steel & Pewter & $1 / 4^{\prime \prime}$ \\
\hline 838 & P330.AFG & Gray & Stainless Steel & Pewter & $1 / 4^{\prime \prime}$ \\
\hline 839 & P430.AFG & Bronze & Stainless Steel & Pewter & $1 / 4^{\prime \prime}$ \\
\hline 840 & P530.AFG & Blue & Stainless Steel & Pewter & $1 / 4 "$ \\
\hline 841 & P630.AFG & Blue-green & Stainless Steel & Pewter & $1 / 4^{\prime \prime}$ \\
\hline 842 & P730.AFG & Azurlite * & Stainless Steel & Pewter & $1 / 4^{\prime \prime}$ \\
\hline 843 & P830.AFG & Evergreen ** & Stainless Steel & Pewter & $1 / 4 "$ \\
\hline 844 & P140.AFG & Clear & Stainless Steel & Pewter & $1 / 4^{\prime \prime}$ \\
\hline 845 & P240.AFG & Green & Stainless Steel & Pewter & $1 / 4^{\prime \prime}$ \\
\hline 846 & P340.AFG & Gray & Stainless Steel & Pewter & $1 / 4^{\prime \prime}$ \\
\hline 847 & P440.AFG & Bronze & Stainless Steel & Pewter & $1 / 4 "$ \\
\hline 848 & P540.AFG & Blue & Stainless Steel & Pewter & $1 / 4^{\prime \prime}$ \\
\hline 849 & P640.AFG & Blue-green & Stainless Steel & Pewter & $1 / 4^{\prime \prime}$ \\
\hline 850 & P740.AFG & Azurlite * & Stainless Steel & Pewter & $1 / 4^{\prime \prime}$ \\
\hline 851 & P840.AFG & Evergreen $* *$ & Stainless Steel & Pewter & $1 / 4^{\prime \prime}$ \\
\hline 852 & S108.AFG & Clear & Stainless Steel & Bright Silver & $1 / 4^{\prime \prime}$ \\
\hline 853 & S208.AFG & Green & Stainless Steel & Bright Silver & $1 / 4 "$ \\
\hline 854 & S308.AFG & Gray & Stainless Steel & Bright Silver & $1 / 4 "$ \\
\hline 855 & S408.AFG & Bronze & Stainless Steel & Bright Silver & $1 / 4^{\prime \prime}$ \\
\hline 856 & S508.AFG & Blue & Stainless Steel & Bright Silver & $1 / 4^{\prime \prime}$ \\
\hline 857 & S608.AFG & Blue-green & Stainless Steel & Bright Silver & $1 / 4 "$ \\
\hline 858 & S708.AFG & Azurlite * & Stainless Steel & Bright Silver & $1 / 4^{\prime \prime}$ \\
\hline 859 & S808.AFG & Evergreen ** & Stainless Steel & Bright Silver & $1 / 4^{\prime \prime}$ \\
\hline 860 & S114.AFG & Clear & Stainless Steel & Bright Silver & $1 / 4^{\prime \prime}$ \\
\hline 861 & S214.AFG & Green & Stainless Steel & Bright Silver & $1 / 4^{\prime \prime}$ \\
\hline 862 & S314.AFG & Gray & Stainless Steel & Bright Silver & $1 / 4^{\prime \prime}$ \\
\hline 863 & S414.AFG & Bronze & Stainless Steel & Bright Silver & $1 / 4^{\prime \prime}$ \\
\hline 864 & S514.AFG & Blue & Stainless Steel & Bright Silver & $1 / 4^{\prime \prime}$ \\
\hline 865 & S614.AFG & Blue-green & Stainless Steel & Bright Silver & $1 / 4^{\prime \prime}$ \\
\hline 866 & S714.AFG & Azurlite * & Stainless Steel & Bright Silver & $1 / 4^{\prime \prime}$ \\
\hline 867 & S814.AFG & Evergreen ** & Stainless Steel & Silver Silver & $1 / 4 "$ \\
\hline 868 & S120.AFG & Clear & Stainless Steel & Bright Silver & $1 / 4 "$ \\
\hline 869 & S220.AFG & Green & Stainless Steel & Bright Silver & $1 / 4^{\prime \prime}$ \\
\hline 870 & S320.AFG & Gray & Stainless Steel & Bright Silver & $1 / 4^{\prime \prime}$ \\
\hline 871 & S420.AFG & Bronze & Stainless Steel & Bright Silver & $1 / 4^{\prime \prime}$ \\
\hline 872 & S520.AFG & Blue & Stainless Steel & Bright Silver & $1 / 4^{\prime \prime}$ \\
\hline 873 & S620.AFG & Blue-green & Stäinless Steel & Bright Silver & $1 / 4^{\prime \prime}$ \\
\hline 874 & S720.AFG & Azurlite * & Stainless Steel & Bright Silver & 1/4" \\
\hline 875 & S820.AFG & Evergreen ** & Stainless Steel & Bright Silver & $1 / 4 "$ \\
\hline 876 & S140.AFG & Clear & Stainless Steel & Bright Silver & $1 / 4^{\prime \prime}$ \\
\hline 877 & S240.AFG & Green & Stainless Steel & Bright Silver & $1 / 4^{\prime \prime}$ \\
\hline 878 & S340.AFG & Gray & Stainless Steel & Bright Silver & $1 / 4^{\prime \prime}$ \\
\hline 879 & S440.AFG & Bronze & Stainless Steel & Bright Silver & $1 / 4^{\prime \prime}$ \\
\hline
\end{tabular}




\section{AFG Industries, Inc.}

\begin{tabular}{|c|c|c|c|c|c|}
\hline (30) & (3) & 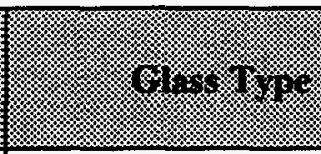 & 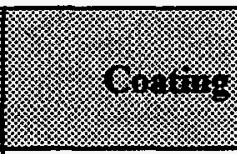 & 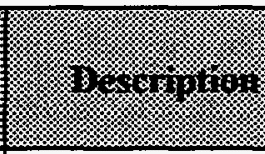 & $\frac{1}{4 y_{1}}$ \\
\hline 880 & S540.AFG & Blue & Stainless Steel & Bright Silver & $1 / 4^{\prime \prime}$ \\
\hline 881 & S640.AFG & Blue-green & Stainless Steel & Bright Silver & $1 / 4 "$ \\
\hline 882 & S740.AFG & Azurlite * & Stainless Steel & Bright Silver & $1 / 4^{\prime \prime}$ \\
\hline 883 & S840.AFG & Evergreen ** & Stainless Steel & Bright Silver & $1 / 4 "$ \\
\hline
\end{tabular}

* Azurlite is a registered trademark of PPG Industries

** Evergreen is a registered trademark of Libbey-Owens-Ford 


\section{Ford Motor Company}

\begin{tabular}{|c|c|c|c|c|c|}
\hline IB: & (4. & 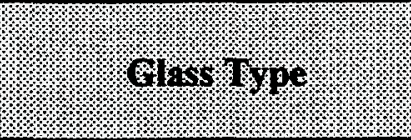 & orating & Description & 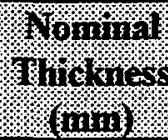 \\
\hline 1300 & CLEAR25.FMC & Clear & & & 2.5 \\
\hline 1301 & CLEAR30.FMC & Clear & & & 3.0 \\
\hline 1302 & CLEAR40.FMC & Clear & & & 4.0 \\
\hline 1303 & CLEAR50.FMC & Clear & & & 5.0 \\
\hline 1304 & CLEAR60.FMC & Clear & & & 6.0 \\
\hline 1305 & GREEN25.FMC & Sunglas ${ }^{\oplus}$ Green & & & 2.5 \\
\hline 1306 & GREEN30.FMC & Sunglas ${ }^{\circ}$ Green & & & 3.0 \\
\hline 1307 & GREEN40.FMC & Sunglas ${ }^{\oplus}$ Green & & & 4.0 \\
\hline 1308 & GREEN50.FMC & Sunglas ${ }^{\theta}$ Green & & & 5.0 \\
\hline 1309 & GREEN60.FMC & Sunglas ${ }^{\circ}$ Green & & & 6.0 \\
\hline 1310 & BRONZE25.FMC & Sunglas ${ }^{\otimes}$ Bronze & & & 2.5 \\
\hline 1311 & BRONZE30.FMC & Sunglas ${ }^{\otimes}$ Bronze & & & 3.0 \\
\hline 1312 & BRONZE40.FMC & Sunglas $^{\otimes}$ Bronze & & & 4.0 \\
\hline 1313 & BRONZE50.FMC & Sunglas ${ }^{\otimes}$ Bronze & & & 5.0 \\
\hline 1314 & BRONZE60.FMC & Sunglas ${ }^{\otimes}$ Bronze & & & 6.0 \\
\hline 1315 & GREY25.FMC & Sunglas ${ }^{\otimes}$ Grey & & & 2.5 \\
\hline 1316 & GREY30.FMC & Sunglas ${ }^{\otimes}$ Grey & & & 3.0 \\
\hline 1317 & GREY40.FMC & Sunglas $^{\otimes}$ Grey & & & 4.0 \\
\hline 1318 & GREY50.FMC & Sunglas $^{\otimes}$ Grey & & & 5.0 \\
\hline 1319 & GREY60.FMC & Sunglas $^{\otimes}$ Grey & & & 6.0 \\
\hline 1320 & BLUE25.FMC & Sunglas ${ }^{*}$ Blue & & & 2.5 \\
\hline 1321 & BLUE30.FMC & Sunglas ${ }^{(2)}$ Blue & & & 3.0 \\
\hline 1322 & BLUE40.FMC & Sunglas ${ }^{8}$ Blue & & & 4.0 \\
\hline 1323 & BLUE50.FMC & Sunglas ${ }^{\otimes}$ Blue & & & 5.0 \\
\hline 1324 & BLUE60.FMC & Sunglas ${ }^{\otimes}$ Blue & & & 6.0 \\
\hline 1325 & REFLGREY.FMC & Sunglas ${ }^{\otimes}$ Reflective Grey & & & 6.0 \\
\hline 1326 & REFLBRZ.FMC & Sunglas ${ }^{\otimes}$ Reflective Bronze & & & 6.0 \\
\hline 1327 & JADEICE.FMC & Sunglas $^{(2)}$ Jadeice $^{\text {rn }}$ & & & 6.0 \\
\hline 1328 & REFLBLUE.FMC & Sunglas ${ }^{\star 0}$ Reflective Blue & & & 6.0 \\
\hline
\end{tabular}


Libbey-Owens-Ford

\begin{tabular}{|c|c|c|c|c|c|}
\hline 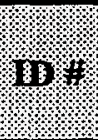 & (1. & 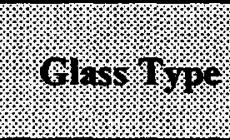 & goating & gescoption & Whiciness \\
\hline 1400 & CLEAR2.LOF & Clear & & & 2.5 \\
\hline 1401 & CLEAR3.LOF & Clear & & & 3 \\
\hline 1402 & CLEAR4.LOF & Clear & & & 4 \\
\hline 1403 & CLEARS.LOF & Clear & & & 5 \\
\hline 1404 & CLEAR6.LOF & Clear & & & 6 \\
\hline 1405 & CLEAR8.LOF & Clear & & & 8 \\
\hline 1406 & CLEAR10.LOF & Clear & & & 10 \\
\hline 1407 & CLEAR12.LOF & Clear & & & 12 \\
\hline 1408 & CLEAR16.LOF & Clear & & & 16 \\
\hline 1409 & CLEAR19.LOF & Clear & & & 19 \\
\hline 1410 & CLEAR25.LOF & Clear & & & 25 \\
\hline 1411 & GREY3.LOF & Grey & & & 3 \\
\hline 1412 & GREY5.LOF & Grey & & & 5 \\
\hline 1413 & GREY6.LOF & Grey & & & 6 \\
\hline 1414 & GREY8.LOF & Grey & & & 8 \\
\hline 1415 & GREY10.LOF & Grey & & & 10 \\
\hline 1416 & GREY12.LOF & Grey & & & 12 \\
\hline 1417 & BRONZ3.LOF & Bronze & & & 3 \\
\hline 1418 & BRONZS.LOF & Bronze & & & 5 \\
\hline 1419 & BRONZ6.LOF & Bronze & & & 6 \\
\hline 1420 & BRONZ8.LOF & Bronze & & & 8 \\
\hline 1421 & BRONZ10.LOF & Bronze & & & 10 \\
\hline 1422 & BRONZ12.LOF & Bronze & & & 12 \\
\hline 1423 & BLGRN6.LOF & Blue-Green & & & 6 \\
\hline 1424 & BLGRN8.LOF & Blue-Green & & & 8 \\
\hline 1425 & BLGRN10.LOF & Blue-Green & & & 10 \\
\hline 1426 & EVGRN3.LOF & Evergreen $^{\mathrm{TM}}$ & & & 3 \\
\hline 1427 & EVGRN5.LOF & Evergreen $^{\mathrm{TM}}$ & & & 5 \\
\hline 1428 & EVGRN6.LOF & Evergreen ${ }^{\mathrm{TM}}$ & & & 6 \\
\hline 1429 & ECLCLR6.LOF & Clear & ECLIPSE @Clear & & 6 \\
\hline 1430 & ECLBLG6.LOF & Blue-Green & ECLIPSE @ Blue-Green & & 6 \\
\hline 1431 & ECLGRY6.LOF & Grey & ECLIPSE \&Grey & & 6 \\
\hline 1432 & ECLBRZ6.LOF & Bronze & ECLIPSE QBronze & & 6 \\
\hline 1433 & ECLGLD6.LOF & Clear & ECLIPSE (1) Gold & & 6 \\
\hline 1434 & MIROPN6.LOF & Grey & MIRROPANE E.P. (8) & & 6 \\
\hline 1435 & LOW-E_2.LOF & Clear & Energy Advantage QLow-E & & 2.5 \\
\hline 1436 & LOW-E_3.LOF & Clear & Energy Advantage (8) Low-E & & 3 \\
\hline 1437 & LOW-E_4.LOF & Clear & Energy Advantage (8) Low-E & & 4 \\
\hline 1438 & LOW-E_5.LOF & Clear & Energy Advantage Low-E & & 5 \\
\hline 1439 & LOW-E_6.LOF & Clear & Energy Advantage \& Low-E & & 6 \\
\hline
\end{tabular}


Southwall Technologies, Inc.

\begin{tabular}{|c|c|c|c|c|c|}
\hline 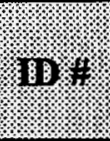 & (4) & 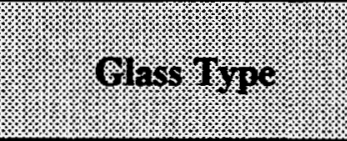 & Yilin & Brescrotion & Whoring \\
\hline 1500 & HM22.SWT & & Heat Mirror ${ }^{\mathrm{TM}} 22$ & Suspended Film & 0.003 \\
\hline 1501 & HM33.SWT & & Heat Mirror ${ }^{\mathrm{TM}} 33$ & Suspended Film & 0.003 \\
\hline 1502 & HM44.SWT & & Heat Mirror ${ }^{\mathrm{TM}} 44$ & Suspended Film & 0.003 \\
\hline 1503 & HM55.SWT & & Heat Mirror ${ }^{\mathrm{TM}} 55$ & Suspended Film & 0.003 \\
\hline 1504 & HM66.SWT & & Heat Mirror ${ }^{\mathrm{TM}} 66$ & Suspended Film & 0.003 \\
\hline 1505 & HM77.SWT & & Heat Mirror ${ }^{\mathrm{TM}} 77$ & Suspended Film & 0.003 \\
\hline 1506 & HM88.SWT & & Heat Mirror ${ }^{\mathrm{TM}} 88$ & Suspended Film & 0.003 \\
\hline 1507 & HMHPR18.SWT & & HM-HPR18 & Suspended Film & 0.003 \\
\hline 1508 & HMHPR28.SWT & & HM-HPR28 & Suspended Film & 0.003 \\
\hline 1509 & HMHPR38.SWT & & HM-HPR38 & Suspended Film & 0.003 \\
\hline 1510 & HMSC75.SWT & & HM-SC75 & Suspended Film & 0.003 \\
\hline 1511 & HMTC88.SWT & & HM-TC88 & Suspended Film & 0.003 \\
\hline 1512 & CSBGRF12.SWT & Blue-Green Reflective & & & $1 / 2^{\prime \prime}$ \\
\hline 1513 & CSBRRF12.SWT & Bronze Reflective & & & $1 / 2^{\prime \prime}$ \\
\hline 1514 & CSCG06.SWT & Carmel Gray & & & $1 / 4^{\prime \prime}$ \\
\hline 1515 & CSCG12.SWT & Carmel Gray & & & $1 / 2 "$ \\
\hline 1516 & CSCLRF12.SWT & Clear Reflective & & & $1 / 2 "$ \\
\hline 1517 & CSGLRF12.SWT & Gold Reflective & & & $1 / 2 "$ \\
\hline 1518 & CSGYRF12.SWT & Gray Reflective & & & $1 / 2 "$ \\
\hline 1519 & CSLESF06.SWT & Low-e Sea Foam Clear & & & $1 / 4^{\prime \prime}$ \\
\hline 1520 & CSLESF12.SWT & Low-e Sea Foam Clear & & & $1 / 2 "$ \\
\hline 1521 & CSMB06.SWT & Mojave Bronze & & & $1 / 4^{\prime \prime}$ \\
\hline 1522 & CSMB12.SWT & Mojave Bronze & & & $1 / 2 "$ \\
\hline 1523 & CSMG06.SWT & Moss Green & & & $1 / 4 "$ \\
\hline 1524 & CSMG12.SWT & Moss Green & & & $1 / 2^{\prime \prime}$ \\
\hline 1525 & CSPB12.SWT & Pacific Blue & & & $1 / 2 "$ \\
\hline 1526 & CSSFC06.SWT & Sea Foam Clear & & & $1 / 4^{\prime \prime}$ \\
\hline 1527 & CSSFC12.SWT & Sea Foam Clear & & & $1 / 2 "$ \\
\hline 1528 & CSSG06.SWT & Sierra Green & & & $1 / 4^{\prime \prime}$ \\
\hline 1529 & CSSG12.SWT & Sierra Green & & & $1 / 2 "$ \\
\hline 1530 & CSTB06.SWT & Tahoe Green & & & $1 / 4^{\prime \prime}$ \\
\hline 1531 & CSTB12.SWT & Tahoe Green & & & $1 / 2 "$ \\
\hline 1532 & CSWWC06.SWT & Water White Crystal & & & $1 / 4^{\prime \prime}$ \\
\hline 1533 & CSWWC12.SWT & Water White Crystal & & & $1 / 2 "$ \\
\hline 1534 & SLCLAZ03.SWT & Azurlite * Glass & Solis Clear & & $1 / 8^{\prime \prime}$ \\
\hline 1535 & SLCLAZ06.SWT & Azurlite * Glass & Solis Clear & & $1 / 4^{\prime \prime}$ \\
\hline 1536 & SLCLBZ03.SWT & Bronze Glass & Solis Clear & & $1 / 8^{\prime \prime}$ \\
\hline 1537 & SLCLBZ06.SWT & Bronze Glass & Solis Clear & & $1 / 4^{\prime \prime}$ \\
\hline 1538 & SLCLCL03.SWT & Clear Glass & Solis Clear & & $1 / 8^{\prime \prime}$ \\
\hline 1539 & SLCLCL06.SWT & Clear Glass & Solis Clear & & $1 / 4^{\prime \prime}$ \\
\hline 1540 & SLCLEG03.SWT & Evergreen ** Glass & Solis Clear & & $1 / 8^{\prime \prime}$ \\
\hline 1541 & SLCLEG06.SWT & Evergreen ** Glass & Solis Clear & & $1 / 4^{\prime \prime}$ \\
\hline 1542 & SLCLFB06.SWT & Blue Glass & Solis Clear & & $1 / 4 "$ \\
\hline 1543 & SLCLGN03.SWT & Green Glass & Solis Clear & & $1 / 8^{\prime \prime}$ \\
\hline 1544 & SLCLGN05.SWT & Green Glass & Solis Clear & & 1/4" \\
\hline 1545 & SLCLGY03.SWT & Gray Glass & Solis Clear & & $1 / 8^{\prime \prime}$ \\
\hline
\end{tabular}


Southwall Technologies, Inc.

\begin{tabular}{|c|c|c|c|c|c|}
\hline 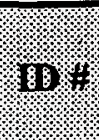 & Hile $14 \%$ ine & 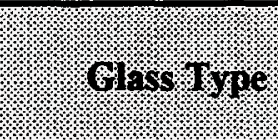 & N11110 & Sesciption & Womping \\
\hline 1546 & SLCLGY06.SWT & Gray Glass & Solis Clear & & $1 / 4 "$ \\
\hline 1547 & SLCLLE06.SWT & Pyrolitic Low-e & Solis Clear & & $1 / 4^{\prime \prime}$ \\
\hline$\overline{1548}$ & SLGYCL03.SWT & Clear Glass & Solis Gray & & $1 / 8^{\prime \prime}$ \\
\hline 1549 & SLGYCL06.SWT & Clear Glass & Solis Gray & & $1 / 4^{\prime \prime}$ \\
\hline 1550 & SOLIS70.SWT & & Solis Clear & Film Alone & .002 \\
\hline
\end{tabular}

* Azurlite is a registered trademark of PPG Industries

** Evergreen is a registered trademark of Libbey-Owens-Ford 
Marcon Coatings

\begin{tabular}{|c|c|c|c|c|c|}
\hline 11111\%. & Milow ranes! & Glasis upe & 9.04ting & Geseriptor & Wouna \\
\hline 1700 & E1-2M-25.MAR & Clear & Low E II & & 2.5 \\
\hline 1701 & E1-2M-3.MAR & Clear & Low E II & & 3.0 \\
\hline 1702 & E1-2M-4.MAR & Clear & Low E II & & 4.0 \\
\hline 1703. & E1-2M-5.MAR & Clear & Low E II & & 5.0 \\
\hline 1704 & E1-2M-6.MAR & Clear & Low E II & & 6.0 \\
\hline 1705 & E1-55-25.MAR & Clear & Southern Low E & & 2.5 \\
\hline 1706 & E1-55-3.MAR & Clear & Southern Low $\mathrm{E}$ & & 3.0 \\
\hline 1707 & E1-55-4.MAR & Clear & Southern Low E & & 4.0 \\
\hline 1708 & E1-55-5.MAR & Clear & Southern Low $\mathrm{E}$ & & 5.0 \\
\hline 1709 & E1-55-6.MAR & Clear & Southern Low E & & 6.0 \\
\hline 1710 & E1-85-25.MAR & Clear & Northern Low E & & 2.5 \\
\hline 1711 & E1-85-3.MAR & Clear & Northern Low E & & 3.0 \\
\hline 1712 & E1-85-4.MAR & Clear & Northern Low $\mathrm{E}$ & & 4.0 \\
\hline 1713 & E1-85-5.MAR & Clear & Northern Low E & & 5.0 \\
\hline 1714 & E1-85-6.MAR & Clear & Northern Low $\mathrm{E}$ & & 6.0 \\
\hline
\end{tabular}




\section{Cardinal IG}

\begin{tabular}{|c|c|c|c|c|c|}
\hline W18 & (niteryme. & Glass Wige & \%.4.7ng. & Bescription & 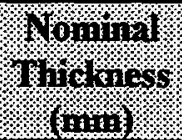 \\
\hline 2000 & CLR 2.CIG & Clear & & & 2.2 \\
\hline 2001 & CLR 3.CIG & Clear & & & 3.0 \\
\hline 2002 & CLR 4. CIG & Clear & & & 4.0 \\
\hline 2003 & CLR 5.CIG & Clear & & & 5.0 \\
\hline 2004 & CLR 6.CIG & Clear & & & 6.0 \\
\hline 2005 & E78 2.CIG & Clear & LoE-78 & & 2.2 \\
\hline 2006 & E78 3.CIG & Clear & LoE-78 & & 3.0 \\
\hline 2007 & E78 4.CIG & Clear & LoE-78 & & 4.0 \\
\hline 2008 & E78 5.CIG & Clear & LoE-78 & & 5.0 \\
\hline 2009 & E78 6.CIG & Clear & LoE-78 & & 6.0 \\
\hline 2010 & EE72 2.CIG & Clear & $\mathrm{LoE}^{2}-72$ & & 2.2 \\
\hline 2011 & EE72 3.CIG & Clear & $\operatorname{LoE}^{2}-72$ & & 3.0 \\
\hline 2012 & EE72 4.CIG & Clear & $\operatorname{LOE}^{2}-72$ & & 4.0 \\
\hline 2013 & EE72 5.CIG & Clear & $\mathrm{LoE}^{2}-72$ & & 5.0 \\
\hline 2014 & EE72 6.CIG & Clear & $\mathrm{LoE}^{2}-72$ & & 6.0 \\
\hline 2015 & SUN45 2.CIG & Clear & LOE SUN-45 & & 2.2 \\
\hline 2016 & SUN45 3.CIG & Clear & LoE SUN-45 & & 3.0 \\
\hline 2017 & SUN45 4.CIG & Clear & LoE SUN-45 & & 4.0 \\
\hline 2018 & SUN45 5.CIG & Clear & LOE SUN 45 & & 5.0 \\
\hline 2019 & SUN45 6.CIG & Clear & LoE SUN-45 & & 6.0 \\
\hline
\end{tabular}




\section{Courtaulds Performance Films}

\begin{tabular}{|c|c|c|c|c|c|}
\hline (10) & Hils 1 grme & 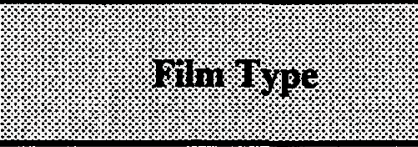 & orting & Bescription & Momingt \\
\hline 2500 & DL30GR.CPF & DL-30GR (Green) SR CDF & & Deluxe & .0015 \\
\hline 2501 & N1020B.CPF & N-1020B (Bronze) SR CDF & & $\mathrm{N}$ (Neutral) & .0015 \\
\hline 2502 & N1020G.CPF & $\mathrm{N}-1020$ (Gray) SR CDF & & $\mathrm{N}$ (Neutral) & .0015 \\
\hline 2503 & N1035B.CPF & N-1035B (Bronze) SR CDF & & $\mathrm{N}$ (Neutral) & .0015 \\
\hline 2504 & N1035G.CPF & N-1035 (Gray) SR CDF & & $\mathrm{N}$ (Neutral) & .0015 \\
\hline 2505 & N1050B.CPF & N-1050B (Bronze) SR CDF & & $\mathrm{N}$ (Neutral) & .0015 \\
\hline 2506 & N1050G.CPF & $\mathrm{N}-1050$ (Gray) SR CDF & & $\mathrm{N}$ (Neutral) & .0015 \\
\hline 2507 & NRM.CPF & NRM-PS2 (Frosty) & & Non-Reflective & .0015 \\
\hline 2508 & NRMB.CPF & NRMB-PS2 (Bronze) & & Non-Reflective & .0015 \\
\hline 2509 & NRMBL.CPF & NRMBL-PS2 (Black) & & Non-Reflective & .0015 \\
\hline 2510 & NRMS.CPF & NRMS-PS2 (Silver & & Non-Reflective & .0015 \\
\hline 2511 & R15B.CPF & R-15B (Bronze) SR CDF & & Reflective & .0015 \\
\hline 2512 & R15BL.CPF & R-15BL (Blue) SR CDF & & Reflective & .0015 \\
\hline 2513 & R15G.CPF & R-15G (Gray) SR CDF & & Reflective & .0015 \\
\hline 2514 & R15GO.CPF & R15GO (Gold) SR CDF & & Reflective & .0015 \\
\hline 2515 & R20.CPF & R-20 (Silver) SR CDF & & Reflective & .0015 \\
\hline 2516 & R30B.CPF & R30B (Bronze) SR CDF & & Reflective & .0015 \\
\hline 2517 & R30G.CPF & R-30G (Gray) SR CDF & & Reflective & .0015 \\
\hline 2518 & R50.CPF & R-50 (Silver) SR CDF & & Reflective & .0015 \\
\hline 2519 & SCL_PS4.CPF & SCL SR-PS4 (Clear) & & Safety & .0015 \\
\hline 2520 & SCL PS7.CPF & SCL SR-PS7 (Clear & & Safety & .0015 \\
\hline 2521 & SCL PS11.CPF & SCL SR-PS11 (Clear) & & Safety & .0015 \\
\hline 2522 & V28.CPF & V-28 SR CDF & & Vista & .0015 \\
\hline 2523 & V30.CPF & V-30 SR CDF & & Vista & .0015 \\
\hline 2524 & V38.CPF & V-38 SR CDF & & Vista & .0015 \\
\hline 2525 & V45.CPF & V-45 SR CDF & & Vista & .0015 \\
\hline 2526 & V58.CPF & V-58 SR CDF & & Vista & .0015 \\
\hline
\end{tabular}




\section{M Corporation}

\begin{tabular}{|c|c|c|c|c|c|}
\hline $10 \%$ & milewane & $40191 \%$ & 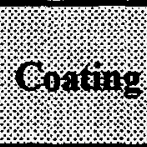 & Descrition & 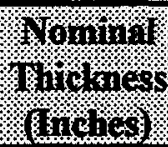 \\
\hline 2700 & P19AR.MMM & P19AR & & Low e, Silver & .0015 \\
\hline 2701 & LE20SIARMMM & LE20SIARL & & Low e, Silver & .0015 \\
\hline 2702 & LE35AMARMMM & LE35AMARL & & Low e, Amber & .0015 \\
\hline 2703 & LE50AMAR.MMM & LE50AMARL & & Low e, Amber & .0015 \\
\hline 2704 & LE30CUARMMM & LE30CUARL & & Low e, Copper & .0015 \\
\hline 2705 & RE15SIX.MMM & RE15SIX & & Sun Control, Silver & .0015 \\
\hline 2706 & P18ARMMM & P18AR & & Sun Control, Silver & .0015 \\
\hline 2707 & RE35AMAR.MMM & RE35AMARL & & Sun Control, Amber & .0015 \\
\hline 2708 & RE20NEARMMM & RE20NEARL & & Sun Control, Neutral & .0015 \\
\hline 2709 & RE35NEARMMM & RE35NEARL & & Sun Control, Neutral & .0015 \\
\hline 2710 & RE50NEARMMM & RE50NEARL & & Sun Control, Neutral & .0015 \\
\hline 2711 & RE70NEARMMM & RE70NEARL & & Sun Control, Neutral & .0015 \\
\hline 2712 & RE20BRARMMM & RE20BRARL & & Sun Control, Bronze & .0015 \\
\hline 2713 & RE35BRAR.MMM & RE35BRARL & & Sun Control, Bronze & .0015 \\
\hline 2714 & RE50BRAR.MMM & RE50BRARL & & Sun Control, Bronze & .0015 \\
\hline 2715 & RE25SLAR.MMM & RE25SLARL & & Sun Control, Slate & .0015 \\
\hline 2716 & RE35SLARMMM & RE35SLARL & & Sun Control, Slate & .0015 \\
\hline 2717 & RE50SLARMMM & RE50SLARL & & Sun Control, Slate & .0015 \\
\hline 2718 & PNTHR5.MMM & PNTHR5 & & Non reflective, Neutral & .0015 \\
\hline 2719 & PNTHR20.MMM & PNTHR20 & & Non reflective, Neutral & .001 \\
\hline 2720 & PNTHR35.MMM & PNTHR35 & & Non reflective, Neutral & .001 \\
\hline 2721 & PNTHR50.MMM & PNTHR50 & & Non reflective, Neutral & .001 \\
\hline 2722 & S35NEARMMM & S35NEARL & & Safety, Neutral & .003 \\
\hline 2723 & SCLAR150.MMM & SCLARL150 & & Safety, Clear & .002 \\
\hline 2724 & SCLAR400.MMM & SCLARL400 & & Safety, Clear & .004 \\
\hline
\end{tabular}




\section{Guardian Industries Glass Products}

\begin{tabular}{|c|c|c|c|c|c|}
\hline $401 \%$ & Hile Yine & Mass & ogating & Deveription & 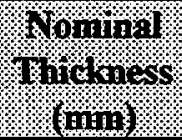 \\
\hline 3100 & PPLUS23C.GRD & Clear & Performance Plus & Low-E & 2.3 \\
\hline 3101 & PPLUS30C.GRD & Clear & Performance Plus & Low-E & 3.0 \\
\hline 3102 & PPLUS30G.GRD & Green & Performance Plus & Low-E & 3.0 \\
\hline 3103 & PPLUS31B.GRD & Bronze & Performance Plus & Low-E & 3.1 \\
\hline 3104 & PPLUS31Y.GRD & Gray & Performance Plus & Low-E & 3.1 \\
\hline
\end{tabular}




\begin{tabular}{|c|c|c|c|c|c|}
\hline $10 \%$ & Mir. & giass mper & goning. & 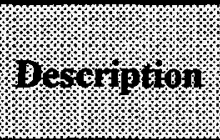 & 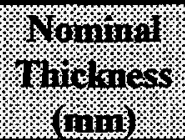 \\
\hline 5001 & STRPH_3.PPG & STARPHIREQ & & & 3.3 \\
\hline 5002 & STRPH_4.PPG & STARPHIREQ & & & 4.0 \\
\hline 5003 & STRPH_5.PPG & STARPHIREQ & & & 5.0 \\
\hline 5004 & STRPH_6.PPG & STARPHIREQ & & & 6.0 \\
\hline 5005 & STRPH 8.PPG & STARPHIREQ & & & 9.0 \\
\hline 5006 & STRPH_10.PPG & STARPHIREQ & & & 10.0 \\
\hline 5007 & STRPH_12.PPG & STARPHIRE@ & & & 12.0 \\
\hline 5008 & CLEAR 2H.PPG & Clear & & & 2.5 \\
\hline 5009 & CLEAR 3.PPG & Clear & & & 3.3 \\
\hline 5010 & CLEAR 4.PPG & Clear & & & 4.0 \\
\hline 5011 & CLEAR 5.PPG & Clear & & & 5.0 \\
\hline 5012 & CLEAR 6.PPG & Clear & & & 6.0 \\
\hline 5013 & CLEAR 8.PPG & Clear & $\div$ & & 8.0 \\
\hline 5014 & CLEAR 10.PPG & Clear & & & 10.0 \\
\hline 5015 & CLEAR 12.PPG & Clear & & & 12.0 \\
\hline 5016 & SOLEX 2H.PPG & SOLEX® & & & 2.5 \\
\hline 5017 & SOLEX_3.PPG & SOLEX® & & & 3.3 \\
\hline 5018 & SOLEX 4.PPG & SOLEXQ & & & 4.0 \\
\hline 5019 & SOLEX_5.PPG & SOLEXQ & & & 5.0 \\
\hline 5020 & SOLEX_6.PPG & SOLEX\& & & & 6.0 \\
\hline 5021 & SOLEX_8.PPG & SOLEX $B$ & & & 8.0 \\
\hline 5024 & SLGRN_2H.PPG & SOLARGREEN@ & & & 2.5 \\
\hline 5025 & SLGRN 3.PPG & SOLARGREEN@ & & & 3.3 \\
\hline 5026 & SLGRN_4.PPG & SOLARGREEN@ & & & 4.0 \\
\hline 5027 & SLGRN_5.PPG & SOLARGREEN@ & & & 5.0 \\
\hline 5028 & SLGRN_6.PPG & SOLARGREEN@ & & & 6.0 \\
\hline 5029 & SLGRN_8.PPG & SOLARGREEN & & & 8.0 \\
\hline 5032 & AZURL 2H.PPG & AZURLITE@ & & & 2.5 \\
\hline 5033 & AZURL_3.PPG & AZURLITEQ & & & 3.3 \\
\hline 5034 & AZURL 4.PPG & AZURLITEQ & & & 4.0 \\
\hline 5035 & AZURL_5.PPG & AZURLITEQ & & & 5.0 \\
\hline 5036 & AZURL_6.PPG & AZURLITEQ & & & 6.0 \\
\hline 5037 & AZURL_8.PPG & AZURLITEQ &. & & 8.0 \\
\hline 5038 & AZURL 10.PPG & AZURLITEQ & & & 10.0 \\
\hline 5040 & SBRNZ 2H.PPG & SOLARBRONZEQ & & & 2.5 \\
\hline 5041 & SBRNZ._3.PPG & SOLARBRONZEQ & & & 3.3 \\
\hline 5042 & SBRNZ_4.PPG & SOLARBRONZEQ & & & 4.0 \\
\hline 5043 & SBRNZ 5.PPG & SOLARBRONZEQ & & & 5.0 \\
\hline 5044 & SBRNZ 6.PPG & SOLARBRONZE@ & & & 6.0 \\
\hline 5045 & SBRNZ_8.PPG & SOLARBRONZE@ & & & 8.0 \\
\hline 5046 & SBRNZ_10.PPG & SOLARBRONZE@ & & & 10.0 \\
\hline 5047 & SBRNZ_12.PPG & SOLARBRONZE@ & & & 12.0 \\
\hline 5049 & SGRAY 3.PPG & SOLARGRAYQ & & & 3.3 \\
\hline 5050 & SGRAY 4.PPG & SOLARGRAYQ & & & 4.0 \\
\hline
\end{tabular}


PPG Industries, Inc.

\begin{tabular}{|c|c|c|c|c|c|}
\hline 1018: & Miev Yame & oryss 490 & Goaning. & Besciolion & Womong \\
\hline 5051 & SGRAY 5.PPG & SOLARGRAY@ & & & 5.0 \\
\hline 5052 & SGRAY_6.PPG & SOLARGRAY@ & & & 6.0 \\
\hline 5053 & SGRAY 8.PPG & SOLARGRAY@ & & & 8.0 \\
\hline 5054 & SGRAY_10.PPG & SOLARGRAY' & & & 10.0 \\
\hline 5055 & SGRAY_12.PPG & SOLARGRAYQ & & & 12.0 \\
\hline 5056 & GRLITE_3.PPG & GRAYLITE@ & & & 3.3 \\
\hline 5057 & GRLITE 6.PPG & GRAYLITE\& & & & 6.0 \\
\hline 5060 & GL20 3.PPG & GL20TM & & & 3.3 \\
\hline 5061 & GL20_6.PPG & GL20 ${ }^{\mathrm{TM}}$ & & & 6.0 \\
\hline 5064 & GL35 3.PPG & GL35TM & & & 3.3 \\
\hline 5065 & GL35 6.PPG & GL35TM & & & 6.0 \\
\hline 5094 & SC_AZ_5.PPG & AZURLITEQ & SOLARCOOL\& & RI Coated & 5.0 \\
\hline 5096 & SC_AZ_6.PPG & AZURLITEQ & SOLARCOOLه & Rl Coated & 6.0 \\
\hline 5102 & SC_BR 3.PPG & SOLARBRONZE@ & SOLARCOOL@ & R1 Coated & 3.3 \\
\hline 5104 & SC_BR 4.PPG & SOLARBRONZE@ & SOLARCOOL@ & R1 Coated & 4.0 \\
\hline 5106 & SC_BR_5.PPG & SOLARBRONZE@ & SOLARCOOL@ & R1 Coated & 5.0 \\
\hline 5108 & SC BR 6.PPG & SOLARBRONZE@ & SOLARCOOL@ & R1 Coated & 6.0 \\
\hline 5110 & SC_BR 8.PPG & SOLARBRONZE@ & SOLARCOOL (\$) & R1 Coated & 8.0 \\
\hline 5112 & SC_BR_10.PPG & SOLARBRONZE@ & SOLARCOOL@ & R1 Coated & 10.0 \\
\hline 5114 & SC_GR_3.PPG & SOLARGRAY@ & SOLARCOOL@ & R1 Coated & 3.3 \\
\hline 5116 & SC_GR_4.PPG & SOLARGRAY@ & SOLARCOOL\& & R1 Coated & 4.0 \\
\hline 5118 & SC_GR 5.PPG & SOLARGRAY@ & SOLARCOOLB & R1 Coated & 5.0 \\
\hline 5120 & SC GR 6.PPG & SOLARGRAY@ & SOLARCOOL $\otimes$ & R1 Coated & 6.0 \\
\hline 5122 & SC_GR_8.PPG & SOLARGRAY@ & SOLARCOOLQ & R1 Coated & 8.0 \\
\hline 5124 & SC_GR_10.PPG & SOLARGRAY@ & SOLARCOOLQ & R1 Coated & 10.0 \\
\hline 5126 & SC_GL_3.PPG & GRAYLITEQ & SOLARCOOLB & R1 Coated & 3.3 \\
\hline 5128 & SC_GL_6.PPG & GRAYLITEO & SOLARCOOLQB & R1 Coated & 6.0 \\
\hline 5140 & S100CL_2.PPG & Clear & SUNGATE®100 & Rl Coated & 2.5 \\
\hline 5142 & S100CL 3.PPG & Clear & SUNGATE@ 100 & R1 Coated & 3.3 \\
\hline 5144 & S100CL 4.PPG & Clear & SUNGATEB 100 & R1 Coated & 4.0 \\
\hline 5146 & S100CL 5.PPG & Clear & SUNGATE@ 100 & R1 Coated & 5.0 \\
\hline 5148 & S100CL 6.PPG & Clear & SUNGATE 8100 & R1 Coated & 6.0 \\
\hline 5150 & S100SX_2.PPG & SOLEX@ & SUNGATE@ 100 & R2 Coated & 2.5 \\
\hline 5151 & S100SX 3.PPG & SOLEX@ & SUNGATE@ 100 & R2 Coated & 3.3 \\
\hline 5152 & S100SX 4.PPG & SOLEX@ & SUNGATE® 100 & R2 Coated & 4.0 \\
\hline 5153 & S100SX 5.PPG & SOLEX® & SUNGATE® 100 & R2 Coated & 5.0 \\
\hline 5154 & S100SX 6.PPG & SOLEX® & SUNGATE@ 100 & R2 Coated & 6.0 \\
\hline 5160 & S100SN 2.PPG & SOLARGREENB & SUNGATE@ 100 & R2 Coated & 2.5 \\
\hline 5161 & S100SN 3.PPG & SOLARGREEN@ & SUNGATE@ 100 & R2 Coated & 3.3 \\
\hline 5162 & S100SN 4.PPG & SOLARGREEN@ & SUNGATE@ 100 & R2 Coated & 4.0 \\
\hline 5163 & S100SN 5.PPG & SOLARGREEN@ & SUNGATE@ 100 & R2 Coated & 5.0 \\
\hline 5164 & S100SN 6.PPG & SOLARGREEN@ & SUNGATE@ 100 & R2 Coated & 6.0 \\
\hline 5170 & S100AZ 2.PPG & AZURLITEQ & SUNGATE® 100 & R2 Coated & 2.5 \\
\hline 5171 & S100AZ 3.PPG & AZURLITE\& & SUNGATE@ 100 & R2 Coated & 3.3 \\
\hline 5172 & S100AZ 4.PPG & AZURLITE@ & SUNGATE\& 100 & R2 Coated & 4.0 \\
\hline
\end{tabular}


PPG Industries, Inc.

\begin{tabular}{|c|c|c|c|c|c|}
\hline BD\% & File Vane & Glass 1 pe & Soq110\% & Bescoption & 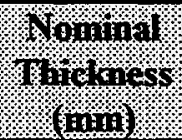 \\
\hline 5173 & S100AZ 5.PPG & AZURLITE@ & SUNGATE\& 100 & R2 Coated & 5.0 \\
\hline 5174 & S100AZ 6.PPG & AZURLITEQ & SUNGATEQ 100 & R2 Coated & 6.0 \\
\hline 5180 & S100BR 2.PPG & SOLARBRONZEQ & SUNGATE\& 100 & R2 Coated & 2.5 \\
\hline 5181 & S100BR 3.PPG & SOLARBRONZE\& & SUNGATE\& 100 & R2 Coated & 3.3 \\
\hline 5182 & S100BR 4.PPG & |SOLARBRONZE@ & SUNGATE@ 100 & R2 Coated & 4.0 \\
\hline 5183 & S100BR 5.PPG & SOLARBRONZE® & SUNGATE\& 100 & R2 Coated & 5.0 \\
\hline 5184 & S100BR 6.PPG & SOLARBRONZE\& & SUNGATEB 100 & R2 Coated & 6.0 \\
\hline 5191 & S100GR 3.PPG & SOLARGRAY@ & SUNGATEB 100 & R2 Coated & 3.3 \\
\hline 5192 & S100GR 4.PPG & SOLARGRAY@ & SUNGATEß 100 & R2 Coated & 4.0 \\
\hline 5193 & S100GR 5.PPG & SOLARGRAY@ & SUNGATEQ 100 & R2 Coated & 5.0 \\
\hline 5194 & S100GR 6.PPG & SOLARGRAY@ & SUNGATE@ 100 & R2 Coated & 6.0 \\
\hline 5220 & S300CL 2.PPG & Clear & - SUNGATEß 300 & R1 Coated & 2.5 \\
\hline 5222 & S300CL 3.PPG & Clear & SUNGATEB 300 & R1 Coated & 3.3 \\
\hline 5224 & S300CL 4.PPG & Clear & SUNGATE@ 300 & R1 Coated & 4.0 \\
\hline 5226 & S300CL 5.PPG & Clear & SUNGATE\& 300 & R1 Coated & 5.0 \\
\hline 5228 & S300CL 6.PPG & Clear & SUNGATE@ 300 & R1 Coated & 6.0 \\
\hline 5240 & S500CL 2.PPG & Clear & SUNGATE@ 500 & R1 Coated & 2.5 \\
\hline 5242 & S500CL 3.PPG & Clear & SUNGATE@ 500 & Rl Coated & 3.3 \\
\hline 5244 & S500CL 4.PPG & Clear & SUNGATE@ 500 & R1 Coated & 4.0 \\
\hline 5246 & S500CL 5.PPG & Clear & SUNGATEB 500 & RI Coated & 5.0 \\
\hline 5248 & S500CL 6.PPG & Clear & SUNGATE@ 500 & R1 Coated & 6.0 \\
\hline
\end{tabular}


Viracon Glass Products

\begin{tabular}{|c|c|c|c|c|c|}
\hline $18 \%$ & Wlo forme & gass yor & Costings. & Bescription & Woringing \\
\hline 6000 & VA113.VIR & Clear & Antique & & 6.0 \\
\hline 6001 & VA118.VIR & Clear & Antique & & 6.0 \\
\hline 6002 & VA122.VIR & Clear & Antique & & 6.0 \\
\hline 6003 & VA135.VIR & Clear & Antique & & 6.0 \\
\hline 6004 & VA213.VIR & Green & Antique & & 6.0 \\
\hline 6005 & VA218.VIR & Green & Antique & & 6.0 \\
\hline 6006 & VA222.VIR & Green & Antique & & 6.0 \\
\hline 6007 & VA235.VIR & Green & Antique & & 6.0 \\
\hline 6008 & VA313.VIR & Gray & Antique & & 6.0 \\
\hline 6009 & VA318.VIR & Gray & Antique & & 6.0 \\
\hline 6010 & VA322.VIR & Gray & Antique & & 6.0 \\
\hline 6011 & VA335.VIR & Gray & Antique & & 6.0 \\
\hline 6012 & VA413.VIR & Bronze & Antique & & 6.0 \\
\hline 6013 & VA418.VIR & Bronze & Antique & & 6.0 \\
\hline 6014 & VA422.VIR & Bronze & Antique & & 6.0 \\
\hline 6015 & VA435.VIR & Bronze & Antique & & 6.0 \\
\hline 6016 & VA513.VIR & Blue & Antique & & 6.0 \\
\hline 6017 & VA518.VIR & Blue & Antique & & 6.0 \\
\hline 6018 & VA522.VIR & Blue & Antique & & 6.0 \\
\hline 6019 & VA535.VIR & Blue & Antique & & 6.0 \\
\hline 6020 & VA613.VIR & Blue-Green & Antique & & 6.0 \\
\hline 6021 & VA618.VIR & Blue-Green & Antique & & 6.0 \\
\hline 6022 & VA622.VIR & Blue-Green & Antique & & 6.0 \\
\hline 6023 & VA635.VIR & Blue-Green & Antique & & 6.0 \\
\hline 6024 & VA713.VIR & Azurlite * & Antique & & 6.0 \\
\hline 6025 & VA718.VIR & Azurlite * & Antique & & 6.0 \\
\hline 6026 & VA722.VIR & Azurlite * & Antique & & 6.0 \\
\hline 6027 & VA735.VIR & Azurlite * & Antique & & 6.0 \\
\hline 6028 & VA813.VIR & Evergreen ** & Antique & & 6.0 \\
\hline 6029 & VA818.VIR & Evergreen ** & Antique & & 6.0 \\
\hline 6030 & VA822.VIR & Evergreen $* *$ & Antique & & 6.0 \\
\hline 6031 & VA835.VIR & Evergreen ** & Antique & & 6.0 \\
\hline 6032 & VB114.VIR & Clear & Blue & & 6.0 \\
\hline 6033 & VB120.VIR & Clear & Blue & & 6.0 \\
\hline 6034 & VB214.VIR & Green & Blue & & 6.0 \\
\hline 6035 & VB220.VIR & Green & Blue & & 6.0 \\
\hline 6036 & VB614.VIR & Blue-Green & Blue & & 6.0 \\
\hline 6037 & VB620.VIR & Blue-Green & Blue & & 6.0 \\
\hline 6038 & VC124.VIR & Clear & Cinnamon & & 6.0 \\
\hline 6039 & VC224.VIR & Green & Cinnamon & & 6.0 \\
\hline 6040 & VC324.VIR & Gray & Cinnamon & & 6.0 \\
\hline 6041 & VC424.VIR & Bronze & Cinnamon & & 6.0 \\
\hline 6042 & VC524.VIR & Blue & Cinnamon & & 6.0 \\
\hline 6043 & VC624.VIR & Blue-Green & Cinnamon & & 6.0 \\
\hline 6044 & VC724.VIR & Azaurlite * & Cinnamon & & 6.0 \\
\hline 6045 & VC824.VIR & Evergreen ** & Cinnamon & & 6.0 \\
\hline 6046 & VE12M.VIR & Clear & Low-E & & 6.0 \\
\hline
\end{tabular}




\begin{tabular}{|c|c|c|c|c|c|}
\hline (1B) & new & GHass Mor. & gogtain & Hescaptingh & 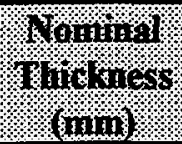 \\
\hline 6047 & VE140.VIR & Clear & Low-E & & 6.0 \\
\hline 6048 & VE152.VIR & Clear & Low-E & & 6.0 \\
\hline 6049 & VE155.VIR & Clear & Low-E & & 6.0 \\
\hline 6050 & VE185.VIR & Clear & Low-E & & 6.0 \\
\hline 6051 & VE22M.VIR & Green & Low-E & & 6.0 \\
\hline 6052 & VE240.VIR & Green & Low-E & & 6.0 \\
\hline 6053 & VE252.VIR & Green & Low-E & & 6.0 \\
\hline 6054 & VE255.VIR & Green & Low-E & & 6.0 \\
\hline 6055 & VE285.VIR & Green & Low-E & & 6.0 \\
\hline 6056 & VE32M.VIR & Gray & Low-E & & 6.0 \\
\hline 6057 & VE340.VIR & Gray & Low-E & & 6.0 \\
\hline 6058 & VE352.VIR & Gray & Low-E & & 6.0 \\
\hline 6059 & VE355.VIR & Gray & Low-E & & 6.0 \\
\hline 6060 & VE385.VIR & Gray & Low-E & & 6.0 \\
\hline 6061 & VE42M. VIR & Bronze & Low-E & & 6.0 \\
\hline 6062 & VE440.VIR & Bronze & Low-E & & 6.0 \\
\hline 6063 & VE452.VIR & Bronze & Low-E & & 6.0 \\
\hline 6064 & VE455.VIR & Bronze & Low-E & & 6.0 \\
\hline 6065 & VE485.VIR & Bronze & Low-E & & 6.0 \\
\hline 6066 & VE52M.VIR & Blue & Low-E & & 6.0 \\
\hline 6067 & VE540.VIR & Blue & Low-E & & 6.0 \\
\hline 6068 & VE552.VIR & Blue & Low-E & & 6.0 \\
\hline 6069 & VE555.VIR & Blue & Low-E & & 6.0 \\
\hline 6070 & VE585.VIR & Blue & Low-E & & 6.0 \\
\hline 6071 & VE62M.VIR & Blue-Green & Low-E & & 6.0 \\
\hline 6072 & VE640.VIR & Blue-Green & Low-E & & 6.0 \\
\hline 6073 & VE652.VIR & Blue-Green & Low-E & & 6.0 \\
\hline 6074 & VE655.VIR & Blue-Green & Low-E & & 6.0 \\
\hline 6075 & VE685.VIR & Blue-Green & Low-E & & 6.0 \\
\hline 6076 & VE72M.VIR & Azurlite * & Low-E & & 6.0 \\
\hline 6077 & VE740.VIR & Azurlite * & Low-E & & 6.0 \\
\hline 6078 & VE752.VIR & Azurlite * & Low-E & & 6.0 \\
\hline 6079 & VE755.VIR & Azurlite * & Low-E & & 6.0 \\
\hline 6080 & VE785.VIR & Azurlite * & Low-E & & 6.0 \\
\hline 6081 & VE82M.VIR & Evergreen ** & Low-E & & 6.0 \\
\hline 6082 & VE840.VIR & Evergreen ** & Low-E & & 6.0 \\
\hline 6083 & VE852.VIR & Evergreen ** & Low-E & & 6.0 \\
\hline 6084 & VE855.VIR & Evergreen ** & Low-E & & 6.0 \\
\hline 6085 & VE885.VIR & Evergreen ** & Low-E & & 6.0 \\
\hline 6086 & VG105.VIR & Clear & Gold & & 6.0 \\
\hline 6087 & VG114.VIR & Clear & Gold & & 6.0 \\
\hline 6088 & VG120.VIR & Clear & Gold & & 6.0 \\
\hline 6089 & VG414.VIR & Bronze & Gold & & 6.0 \\
\hline 6090 & VG420.VIR & Bronze & Gold & & 6.0 \\
\hline 6091 & VS108.VIR & Clear & Stainless Steel & & 6.0 \\
\hline 6092 & VS114.VIR & Clear & Stainless Steel & & 6.0 \\
\hline 6093 & VS120.VIR & Clear & Stainless Steel & & 6.0 \\
\hline
\end{tabular}




\section{Viracon Glass Products}

\begin{tabular}{|c|c|c|c|c|c|}
\hline $10 \%$ : & Milevlane. & 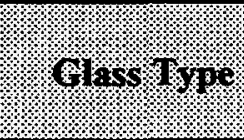 & Soring & Hescinition & Honina \\
\hline 6094 & VS208.VIR & Green & Stainless Steel & & 6.0 \\
\hline 6095 & VS214.VIR & Green & Stainless Steel & & 6.0 \\
\hline 6096 & VS220.VIR & Green & Stainless Steel & & 6.0 \\
\hline 6097 & VS308.VIR & Gray & Stainless Steel & & 6.0 \\
\hline 6098 & VS314.VIR & Gray & Stainless Steel & & 6.0 \\
\hline 6099 & VS320.VIR & Gray & Stainless Steel & & 6.0 \\
\hline 6100 & VS408.VIR & Bronze & Stainless Steel & & 6.0 \\
\hline 6101 & VS414.VIR & Bronze & Stainless Steel & & 6.0 \\
\hline 6102 & VS420.VIR & Bronze & Stainless Steel & & 6.0 \\
\hline 6103 & VS508.VIR & Blue & Stainless Steel & & 6.0 \\
\hline 6104 & VS514.VIR & Blue & Stainless Steel & & 6.0 \\
\hline 6105 & VS520.VIR & Blue & Stainless Steel & & 6.0 \\
\hline 6106 & VS608.VIR & Blue-Green & Stainless Steel & & 6.0 \\
\hline 6107 & VS614.VIR & Blue-Green & Stainless Steel & & 6.0 \\
\hline 6108 & VS620.VIR & Blue-Green & Stainless Steel & & 6.0 \\
\hline 6109 & VS708.VIR & Azurlite * & Stainless Steel & & 6.0 \\
\hline 6110 & VS714.VIR & Azurlite * & Stainless Steel & & 6.0 \\
\hline 6111 & VS720.VIR & Azurlite * & Stainless Steel & & 6.0 \\
\hline 6112 & VS808.VIR & Evergreen ** & Stainless Steel & & 6.0 \\
\hline 6113 & VS814.VIR & Evergreen ** & Stainless Steel & & 6.0 \\
\hline 6114 & VS820.VIR & Evergreen $* *$ & Stainless Steel & & 6.0 \\
\hline 6115 & VT120.VIR & Clear & Titanium & & 6.0 \\
\hline 6116 & VT130.VIR & Clear & Titanium & & 6.0 \\
\hline 6117 & VT135.VIR & Clear & Titanium & & 6.0 \\
\hline 6118 & VT140.VIR & Clear & Titanium & & 6.0 \\
\hline 6119 & VT220.VIR & Green & Titanium & & 6.0 \\
\hline 6120 & VT230.VIR & Green & Titanium & & 6.0 \\
\hline 6121 & VT235.VIR & Green & Titanium & & 6.0 \\
\hline 6122 & VT240.VIR & Green & Titanium & & 6.0 \\
\hline 6123 & VT320.VIR & Gray & Titanium & & 6.0 \\
\hline 6124 & VT330.VIR & Gray & Titanium & & 6.0 \\
\hline 6125 & VT335.VIR & Gray & Titanium & & 6.0 \\
\hline 6126 & VT340.VIR & Gray & Titanium & & 6.0 \\
\hline 6127 & VT420.VIR & Bronze & Titanium & & 6.0 \\
\hline 6128 & VT430.VIR & Bronze & Titanium & & 6.0 \\
\hline 6129 & VT435.VIR & Bronze & Titanium & & 6.0 \\
\hline 6130 & VT440.VIR & Bronze & Titanium & & 6.0 \\
\hline 6131 & VT520.VIR & Blue & Titanium & & 6.0 \\
\hline 6132 & VT530.VIR & Blue & Titanium & & 6.0 \\
\hline 6133 & VT535.VIR & Blue & Titanium & & 6.0 \\
\hline 6134 & VT540.VIR & Blue & Titanium & & 6.0 \\
\hline 6135 & VT620.VIR & Blue-Green & Titanium & & 6.0 \\
\hline 6136 & VT630.VIR & Blue-Green & Titanium & & 6.0 \\
\hline 6137 & VT635.VIR & Blue-Green & Titanium & & 6.0 \\
\hline 6138 & VT640.VIR & Blue-Green & Titanium & & 6.0 \\
\hline 6139 & VT720.VIR & Azurlite * & Titanium & & 6.0 \\
\hline
\end{tabular}




\section{Viracon Glass Products}

\begin{tabular}{|c|c|c|c|c|c|}
\hline $110 \%$ & Wllet Wme & 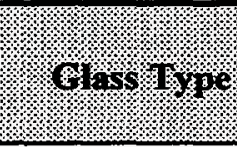 & \%ormg & Mescholion & 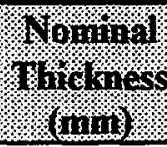 \\
\hline 6140 & VT730.VIR & Azurlite * & Titanium & & 6.0 \\
\hline 6141 & VT735.VIR & Azurlite * & Titanium & & 6.0 \\
\hline 6142 & VT740.VIR & Azurlite * & Titanium & & 6.0 \\
\hline 6143 & VT820.VIR & Evergreen ** & Titanium & & 6.0 \\
\hline 6144 & VT830.VIR & Evergreen ** & Titanium & & 6.0 \\
\hline 6145 & VT835.VIR & Evergreen ** & Titanium & & 6.0 \\
\hline 6146 & VT840.VIR & Evergreen ** & Titanium & & 6.0 \\
\hline
\end{tabular}

* Azurlite is a registered trademark of PPG Industries

** Evergreen is a registered trademark of Libbey-Owens-Ford 
